\title{
A Note on a hyper-cubic Mahler measure and associated Bessel integral.
}

\author{
ML Glasser $\dagger$ \\ $\dagger$ Department of Physics, Clarkson University, Potsdam
} NY 13699-5820, USA

Dedicated to Fa Yueh Wu in recognition of his 80-th birthday

\begin{abstract}
The Mahler measure for the $\mathrm{n}$-variable polynomial $k+\sum\left(x_{j}+1 / x_{j}\right)$ is reduced to a single integral of the $n-t h$ power of the modified Bessel Function $I_{0}$. Several special cases are examined in detail.
\end{abstract}

PACS numbers: 02.30.Gp, 05.50.+q

Submitted to: J. Phys. A: Math. Gen.

\section{Introduction}

Interest in the Mahler measure

$$
m(P)=\int_{0}^{1} d t_{1} \cdots \int_{0}^{1} d t_{n} \ln \left|P\left(e^{2 \pi i t_{1}}, \cdots, e^{2 \pi i t_{n}}\right)\right|
$$

of a polynomial $P\left(x_{1}, \cdots, x_{n}\right)$ has grown since Deninger[1] and Boyd[2] formulated a number of conjectures connecting them with the $L$-functions of an important class of elliptic curves. More recently, Bertin[3] and Rogers[4,5] have extended this connection to hypergeometric identities and multi-dimensional lattice sums. For restricted ranges of the coefficients of certain $P, m(P)$ reduces to a variety of entropic integrals of interest in statistical physics, such as the spanning tree generating functions, studied extensively by Wu and his co-workers[6,7,8,9], as well as their derivatives, the corresponding lattice Green functions[10].

The aim of this note is to examine the Mahler measure of the hyper-cubic "polynomial"

$$
k+\sum_{j=1}^{n}\left(x_{j}+1 / x_{j}\right) .
$$

With $k=2 z, z \geq n$, after a simple change of integration variables, (1) becomes

$$
m(P)=\ln 2+J_{n}(z)
$$




$$
J_{n}(z)=\frac{1}{\pi^{n}} \int_{0}^{\pi} d x_{1} \cdots \int_{0}^{\pi} d x_{n} \ln \left[z \pm \sum_{j=1}^{n} \cos x_{j}\right] .
$$

(The sign is irrelevant). In 1987 the case $J_{3}(3)$ was first studied by A. Rosengren[11] and subsequently Joyce and Zucker[12] investigated $J_{d}(d)$ by reducing it to the single integral

$$
J_{d}(d)=\int_{0}^{\infty}\left[e^{-t}-e^{-t d} I_{0}^{d}(t)\right] \frac{d t}{t} .
$$

By means of an asymptotic expansion Joyce and Zucker could approximate the integral (4) and obtain $J_{d}(d)$ to 50 place accuracy for $d=2, \cdots, 10$. In addition they worked out 15 terms of the asymptotic expansion for $d \rightarrow \infty$. The principal result of this note is the derivation of an expression for $J_{n}(z)$ similar to (4) for arbitrary $z>n$ and to explore some consequences.

\section{Calculation}

We start with the integral representation

$$
\ln A=-\gamma-A \int_{0}^{\infty} d x \ln x e^{-A x}
$$

Then, with $A=z+\sum \cos x_{j}$, the familiar representation

$$
I_{0}(t)=\frac{1}{\pi} \int_{0}^{\pi} d x e^{ \pm t \cos x}
$$

and the fact that $I_{0}^{\prime}(t)=-I_{1}(t)$, we have

$$
J_{n}(z)=-\gamma-\int_{0}^{\infty} d x \ln x e^{-z x} I_{0}^{n-1}(x)\left[z I_{0}(x)+n I_{1}(x)\right] .
$$

Being careful about the cancellation of the divergences at the lower limit, the second integral can be integrated by parts and recombined with the first to yield

$$
J_{n}(z)=-\gamma+\int_{0}^{\infty} \frac{d x}{x}\left[\Theta(1-x)-e^{-z x} I_{0}^{n}(x)\right]=\int_{0}^{\infty} \frac{d x}{x}\left[e^{-x}-e^{-z x} I_{0}^{n}(x)\right],
$$

where $\Theta$ denotes the unit step function. Equation (8) is valid for $z \geq n \geq 0$ and is consistent with Joyce and Zucker's expression (4) since

$$
\int_{0}^{1} \frac{1-e^{-x}}{x} d x-\int_{1}^{\infty} \frac{e^{-x}}{x} d x=\gamma
$$

\section{Results and conclusions}

By comparing (8) with the known values of $J_{n}(z)$ for $n=1,2$ we obtain several, apparently, new Bessel integrals:

$$
\begin{gathered}
\int_{0}^{\infty} \frac{d x}{x}\left[\Theta(x-1)-e^{-z x} I_{0}(x)\right]=\gamma+\ln \left(\frac{z+\sqrt{z^{2}-1}}{2}\right) \\
\int_{0}^{\infty} \frac{d x}{x}\left[e^{-x}-e^{-z x} I_{0}^{2}(x)\right]=\ln (z)-\frac{1}{2 z^{2}}{ }_{4} F_{3}\left(1,1, \frac{3}{2}, \frac{3}{2} ; 2,2,2 ; \frac{4}{z^{2}}\right)
\end{gathered}
$$


Note that

$$
\begin{gathered}
\int_{0}^{\infty} d x \ln x e^{-z x}\left[z I_{0}^{2}(x)+2 I_{0}(x) I_{1}(x)\right]= \\
\ln (1 / z)-\gamma+\frac{1}{2 z^{2}}{ }_{4} F_{3}\left(1,1, \frac{3}{2}, \frac{3}{2} ; 2,2,2 ; \frac{4}{z^{2}}\right) .
\end{gathered}
$$

$$
\begin{gathered}
{ }_{4} F_{3}\left(1,1, \frac{3}{2}, \frac{3}{2} ; 2,2,2 ; z\right)= \\
\frac{16}{\pi z} \int_{0}^{\sqrt{z}} \frac{d u}{u}[\mathbf{K}(u)-\mathbf{K}(0)] .
\end{gathered}
$$

Also ${ }_{4} F_{3}(1,1,3 / 2,3 / 2 ; 2,2,2 ; 1)=16(\pi \ln 2-2 \mathbf{G}) / \pi$.

Next, from the techniques introduced in Ref.[13] one finds

$$
\begin{gathered}
\int_{0}^{\infty} e^{-z t} I_{0}^{3}(t) d t= \\
\frac{3(r+2 z)}{10\left(z^{2}-9\right)} F\left[-\frac{16(2 z(r-z)+9}{\left(z^{2}-9\right)^{2}}\right]-\frac{\left(z^{2}+15\right) r-2 z^{3}+18 z}{5\left(\left(z^{2}-9\right)\left(z^{2}+3\right)\right.} F\left[-\frac{32 z(r-z)+144}{(z(r-z)+3)^{4}}\right] \\
+\frac{18(r-2 z)}{5\left(z^{2}-9\right)\left(z^{2}+3\right)}
\end{gathered}
$$

where $r=\sqrt{z^{2}-9}$ and $F[\xi]={ }_{3} F_{2}(1 / 4,1 / 2,3 / 4 ; 1,1 ; \xi)$. By integrating (14) with respect $z$ from $z=5$, where the second term of (14) vanishes and the first term simplifies considerably, to $z$ (this was carried out using Mathematica), we arrive at the closed-form expression

$$
\begin{gathered}
J_{3}(z)=\int_{0}^{\infty} \frac{d x}{x}\left[e^{-x}-e^{-z x} I_{0}^{3}(x)\right]= \\
-\frac{1}{5}\left\{\ln \left[\frac{2 R_{0}}{\left(z^{2}+3\right)^{3}}\right]+\frac{9}{8} \frac{R_{1}^{3}}{R_{2}^{4}}{ }_{5} F_{4}\left(1,1,5 / 4,3 / 2,7 / 4 ; 2,2,2,2 ; 16 \frac{R_{1}^{3}}{R_{2}^{4}}\right)\right. \\
\left.+\frac{3}{8} \frac{R_{1}}{R_{3}^{4}}{ }_{5} F_{4}\left(1,1,5 / 4,3 / 2,7 / 4 ; 2,2,2,2 ; 16 \frac{R_{1}}{R_{3}^{4}}\right)\right\},
\end{gathered}
$$

where

$$
\begin{gathered}
R_{0}=z^{3}-5 z-\left(z^{2}-1\right) \sqrt{z^{2}-3} \\
R_{1}=2 z^{2}-9-2 z \sqrt{z^{2}-9} \\
R_{2}=z^{2}-9-z \sqrt{z^{2}-9} \\
R_{3}=z^{2}-3-z \sqrt{z^{2}-9}
\end{gathered}
$$

For $3 \leq z \leq 5$ the use of (15) requires the analytic continuation of the hypergeometric function which is examined in Appendix A; for $z>5$ (15) is correct as stated. An equivalent expression appears in the work of Guttmann and Rogers[16]. The value (15) is a companion to Joyce's expressions[15] for the FCC lattice.

Can one proceed in this way? In view of the work by Glasser and Guttmann [17] concerning

$$
\int_{0}^{\infty} e^{-z x} I_{0}^{4}(x) d x
$$

which includes its differential equation and series expansions, $n=3$ is probably as far as one can go in terms of known hypergeometric functions defined by a single series. 


\section{Acknowledgments}

The author thanks Tony Guttmann, John Zucker and Mathew Rogers for substantial help with this project.

\section{Appendix A. Analytic continuation of ${ }_{5} F_{4}(1,1,5 / 4,3 / 2,7 / 4 ; 2,2,2,2 ; z)$}

By examining the respective series, it is clear that

$$
{ }_{5} F_{4}(1,1,5 / 4,3 / 2,7 / 4 ; 2,2,2,2 ; z)=\frac{1}{z} \int_{0}^{z} d t{ }_{4} F_{3}(1,5 / 4,3 / 2,7 / 4 ; 2,2,2 ; t) .
$$

Similarly,

$$
{ }_{4} F_{3}(1,5 / 4,3 / 2,7 / 4 ; 2,2,2 ; t)=\frac{1}{t} \int_{0}^{t} d u{ }_{3} F_{2}(5 / 4,3 / 2,7 / 4 ; 2,2 ; u) .
$$

However,

$$
{ }_{3} F_{2}(5 / 4,3 / 2,7 / 4 ; 2,2 ; u)=\frac{32}{3} \frac{d}{d u}{ }_{3} F_{2}(1 / 4,1 / 2,3 / 4 ; 1,1 ; u) .
$$

Since,

$$
\begin{aligned}
& { }_{3} F_{2}(a, b, a+1 / 2 ; 1,2 a+b ; z) \\
& =\left[\frac{2}{z}(1-\sqrt{1-z})\right]^{2 a}{ }_{3} F_{2}\left(2 a, 2 a, 1-b ; 1,2 a+b ; 1-\frac{2}{z}(1-\sqrt{1-z})\right),
\end{aligned}
$$

by combining these relations, after integrating by parts and noting that

$$
{ }_{3} F_{2}(1 / 2,1 / 2,1 / 2 ; 1,1 ; z)=\frac{4}{\pi^{2}} \mathbf{K}^{2}\left(\sqrt{\frac{1-\sqrt{1-z}}{2}}\right),
$$

after change of integration variable, one finds

$$
\begin{gathered}
{ }_{5} F_{4}(1,1,5 / 4,3 / 2,7 / 4 ; 2,2,2,2 ; z)= \\
\frac{256}{3 \pi^{2} z} \int_{0}^{\alpha(z)} \frac{d t}{t} \frac{\left(1-6 t^{2}+t^{4}\right)}{1-t^{4}}\left[\left(1+t^{2}\right) \mathbf{K}^{2}(t)-\mathbf{K}^{2}(0)\right] \\
\alpha(z)=\sqrt{\frac{\sqrt{2}-\sqrt{1+\sqrt{1-z}}}{\sqrt{2}+\sqrt{1+\sqrt{1-z}}}} .
\end{gathered}
$$

This shows analyticity in the $z$-plane cut along the positive real axis for $z \geq 1$.

\section{References}

[1] C. Deninger, J. Amer. Math. Soc.10,259-281 (1997)

[2] D.W. Boyd, Experimental Math. 7, 37-82 (1998)

[3] M.J. Bertin, J. Num. Theory 128, 2890-2913 (2008) 
[4] M. Rogers, Ramanujan J. 18,327-340(2011)

[5] M. Rogers, IMRN 17,4027-4058 (2011)

[6] F.Y. Wu, J.Phys. A:Math. Gen.10, L113-L115(1977)

[7] R. Shrock and F.Y. Wu, J. Phys. A:Math.Gen.38,3881-3902(2007)

[8] F.Y. Wu and M.L. Glasser, Ramanujan J. 10, 205-215(2005)

[9] M.L. Glasser and G. Lamb, J. Phys. A:Math. Gen.38, L471-L473(2005)

[10] I.J. Zucker, J. Stat. Phys 145, 591-612(2011)

[11] A. Rosegren, J. Phys. A:Math.Gen.20, L993-L927 (1987)

[12] G.S. Joyce and I.J. Zucker, J.Phys. A:Math.Gen.34, 7349-7354 (2001)

[13] M.L. Glasser and E. Montaldi, Physical Rev. E48, R2340-R2342 (1993)

[14] G.S. Joyce, Phil. Trans. Roy. Soc. London A273, 573-610(1973)

[15] G.S. Joyce, J. Phys. A: Math. Theor.45, 285001(2012).

[16] A.J. Guttmann and M. Rogers, Arxiv:math-phys.1207.2815v1 (2012);

[17] M.L. Glasser and A.J. Guttmann, J. Phys. A:Math. Gen.27, 7011-7014 (1974) 